\title{
Experimental Validation of Source Seeking with a Switching Strategy
}

\author{
Wencen Wu and Fumin Zhang
}

\begin{abstract}
We design a switching strategy for a group of robots to search for a local minimum of an unknown noisy scalar field. Starting with individual exploration, the robots switch to cooperative exploration only when they are not able to locate the field minimum based on the information collected individually. In order to test and demonstrate the switching strategy in real-world environment, we implement the switching strategy on a multi-robot test-bed. The behaviors of a group of robots are compared when different parameters for exploration are adopted. Especially, we observe the effect of memory lengths on the switching behaviors as predicted by theoretical results. The experimental results also justify the effects of different formation sizes and noise attenuation levels on the performance of the cooperative $H_{\infty}$ filter that are utilized in the cooperative exploration phase.
\end{abstract}

\section{INTRODUCTION}

The cooperative exploration problem concerns how to deploy a group of mobile robots to explore an unknown scalar field efficiently and adaptively [1]-[4]. In some scenarios, a cooperative group of robots are expected to perform better than a single robot [5]. However, increasing the number of robots results in rising costs, communication delay, and computational complexity. Therefore, the exploring behavior of each robot does not have to be fixed. As observed in nature by biologists, fish in a group only exchange information with others when they can not gather reliable information individually [6]. Inspired by the grouping behaviors of fishes, we proposed a switching strategy for a networked group of robots to search for a local minimum of an unknown field in [7]. Compared to other source seeking algorithms [1], [8][10], our strategy offers a novel aspect that focuses on the switching between individual exploration and cooperative exploration. Our results can be combined with the exploration strategy reviewed to allow balances between individual and cooperative behaviors.

In this paper, we discuss the implementation of the switching strategy and develop a multi-robot exploration testbed to justify the strategy in real-world environments. The basic idea of the switching strategy is that the robots keep exploring the field individually if they can be guaranteed to find a local minimum. They communicate and switch to cooperative exploration only when the convergence rates are low. The switching condition from individual exploration to cooperative exploration is derived based on the Razumikhin theorem, which is developed for verifying the stability of

The research work is supported by ONR grants N00014-08-1-1007, N00014-09-1-1074, and N00014-10-10712 (YIP), and NSF grants ECCS0841195 (CAREER), CNS-0931576, and ECCS-1056253. Wencen Wu and Fumin Zhang are with the School of Electrical and Computer Engineering, Georgia Institute of Technology, Atlanta, GA, 30332, USA wwencen3, fumin@gatech.edu time-delay systems [11] [12]. We extend the switching condition so that the robots can locate a nonzero local minimum of an unknown field. The switching condition from cooperative exploration to individual exploration is based on the change of the signal-to-noise ratio (SNR) of the field. A cooperative $H_{\infty}$ filter is constructed to provide estimates of the field value and the field gradient at the center of the formation formed by the robots after they switch to cooperative exploration. Unlike Kalman filters which assume noises to be Gaussian, $H_{\infty}$ filters can deal with noises with unknown properties [13]-[15], which is more realistic. The convergence and feasibility of the cooperative $H_{\infty}$ filter regarding a symmetric formation are proved.

Computer simulation presented in [7] is a good way to verify the exploring strategy. However, experimental validation on real robots is also important since there are real-world uncertainties and variations that are not able to be considered beforehand. For our exploration algorithm, it is hard to produce realistic noisy field that is non-Gaussian through simulation, which makes experimental effort necessary. In addition, experiments will validate the theoretical bounds for the noise attenuation level in the cooperative $H_{\infty}$ filter, so that understandings of the conservativeness of such bounds for real systems can be obtained. Furthermore, experiments can verify that the switching conditions we developed are robust to communication delays in real life systems. Finally, experiments establish connections between biological systems and engineering systems in that the experimental data collected in either cases can be directly compared.

We develop a multi-robot exploration test-bed in a laboratory environment which can be utilized for testing different cooperative exploration algorithms. The switching strategy for seeking for a local minimum is implemented on the testbed. Other Multi-robot test-beds have been designed [16] [18] to verify and validate different exploration algorithms. In our experiments, we conduct several trials with respect to different memory lengths that are assigned to the robots, different formation sizes, and various noise attenuation levels for the cooperative $H_{\infty}$ filter. We verify the influence of the memory length on the exploration behavior of the robots and justify the effects of different formation sizes and noise attenuation levels on the performance of the cooperative $H_{\infty}$ filter.

The rest of the paper is organized as follows. In section II, we introduce the switching strategy that are implemented on the test-bed. In section III, we first describe the configuration of the multi-robot exploration test-bed. Then we perform the experiments and present the results. Concluding remarks are presented in section IV. 


\section{The SWitching StRATEgy}

In this section, we describe the switching strategy for a group of robots to search for local minimums of an unknown field developed in [7]. Suppose that $z(\mathbf{r}), \mathbf{r} \in \mathbb{R}^{2}$ is an unknown smooth scalar field that is perturbed by unknown noises. We assume that each robot can only take one measurement of the field at each time instance.

\section{A. Individual exploration}

Denote the position of a robot at the $k$ th time step as $\mathbf{r}_{k}$, the measurement taken by the robot as $p_{k}$ and the true field value at position $\mathbf{r}_{k}$ as $z\left(\mathbf{r}_{k}\right)=z_{k}$. In our previous paper [7], we developed the switching condition from individual exploration to cooperative exploration based on the Razumikhin theorem [11]. If the switching condition is not violated, the convergence to a local minimum, which is assumed to be zero, is guaranteed. However, given an unknown field, we usually have no information about the field value. Therefore, we extend the switching condition so that the robots can converge to a nonzero local minimum if the switching condition is not violated.

We first restate the Razumikhin Theorem for the asymptotic stability of time-delay systems [11] without proof.

Theorem 1: (Razumikhin Theorem) Given a system $\dot{x}(t)=f\left(t, x_{t}\right)$ where $x \in \mathbb{R}^{n}$ and $x_{t}$ represents the time delayed system trajectory, suppose $f: \mathbb{R} \times C \rightarrow \mathbb{R}^{n}$ takes bounded subsets of $C$ into bounded subsets of $\mathbb{R}^{n}$. Suppose $\alpha_{1}, \alpha_{2}, w: \mathbb{R}_{+} \rightarrow \mathbb{R}_{+}$are continuous nondecreasing functions, $\alpha_{1}(u)>0, \alpha_{2}(u)>0$ and $w(u)>0$ for $u>0$. Suppose $\alpha_{1}(0)=\alpha_{2}(0)=0$ and $\alpha_{2}$ is strictly increasing. Suppose there exists a continuous nondecreasing function $g(u)>u$ for $u>0$. If there exists a continuous differentiable function $V: \mathbb{R} \times \mathbb{R}^{n} \rightarrow \mathbb{R}$ such that

$$
\alpha_{1}(\|x\|) \leq V(t, x) \leq \alpha_{2}(\|x\|), \forall t \in \mathbb{R}, x \in \mathbb{R}^{n},
$$

and the derivative of $V$ along the solution $x(t)$ satisfies

$$
\dot{V}(t, x(t)) \leq-w(\|x(t)\|),
$$

whenever $V(t+\theta), x(t+\theta)) \leq g(V(t, x(t)))$ for all $\theta \in[-r, 0]$, then the equilibrium $x(t)=0$ of the system is asymptotically stable.

For discrete time systems with delays, condition (2) becomes [12]

$$
V(k+1, x(k+1))-V(k, x(k)) \leq-w(\|x(k)\|),
$$

whenever $V(k+\theta, x(k+\theta)) \leq g(V(k, x(k)))$ for all $\theta \in[-r, 0]$. Based on the Razumikhin theorem, we have the following proposition.

Proposition 1: Suppose the field value $z_{k}$ satisfy $z_{\min } \leq z_{k} \leq z_{\max }, \forall k$. Let $\bar{p}_{k}=\max _{s \in[-r, 0]} p_{k+s}$ where $r \in \mathbf{Z}^{+}$ and $p_{k}$ is the measurement at the time instance $k$. If $p_{k+1}-p_{k} \leq-\rho p_{k}+\rho z_{\text {min }}$ whenever $(1+\varepsilon) p_{k} \geq \bar{p}_{k}+\varepsilon z_{\text {min }}$ where $\rho, \varepsilon>0$ are infinitesimal constants, then $p_{k}$ will converge to $z_{\min }$ as $k \rightarrow \infty$.

Proof: Define a new variable $y_{k}=p_{k}-z_{\min } \geq 0$, so we have $y_{\max }=z_{\max }-z_{\min }$ and $0 \leq y_{k} \leq y_{\max }$. Let $V\left(y_{k}\right)=$ $y_{k} \in\left[0, y_{\max }\right]$. Define $\bar{y}_{k}=\max _{s \in[-r, 0]} y_{k+s}$. If we choose $g\left(V\left(y_{k}\right)\right)=(1+\varepsilon) y_{k}$ where $\varepsilon>0$ is an infinitesimal constant, then the condition $V\left(\bar{y}_{k}\right) \leq g\left(V\left(y_{k}\right)\right)$ in the Razumikhin theorem becomes $\max _{s \in[-r, 0]} y_{k+s} \leq(1+\varepsilon) y_{k}$, which can be written as $\max _{s \in[-r, 0]}\left(p_{k+s}-z_{\min }\right) \leq(1+\varepsilon)\left(p_{k}-z_{\min }\right)$. If we further simplify the condition, we can obtain: $\bar{p}_{k}+\varepsilon z_{\min } \leq$ $(1+\varepsilon) p_{k}$. We also have $V\left(y_{k+1}\right)-V\left(y_{k}\right)=p_{k+1}-p_{k}$. If we choose $w\left(y_{k}\right)=\rho y_{k}=\rho p_{k}-\rho z_{\min }$ where $\rho$ is another infinitesimal constant, then according to the Razumikhin Theorem on asymptotic stability, if for all $k \in[r, \infty)$, the measurements satisfy $p_{k+1}-p_{k} \leq-\rho p_{k}-\rho z_{\min }$ whenever $(1+\varepsilon) p_{k} \geq \bar{p}_{k}+\varepsilon z_{\min }$, then $y_{k}$ converges to 0 as $k \rightarrow \infty$, which implies $p_{k}$ converges to $z_{\min }$ as $k \rightarrow \infty$.

Based on the above proposition, we have the following exploration algorithm for individual exploration.

Algorithm 1: Suppose a robot is searching for a local minimum of an unknown field where the field value satisfies $z_{\min } \leq z_{k} \leq z_{\max }$. Let $\bar{p}_{k}=\max _{s \in[-r, 0]} p_{k+s}$ where $r$ is the memory length of the robot. At each time step $k \geq r$,

(1) The robot takes a measurement of the field $p_{k}$. Then estimates the field gradient $\nabla z_{k}$ by solving the equations $\frac{p_{k}-p_{k-1}}{\left\|\mathbf{r}_{k}-\mathbf{r}_{k-1}\right\|}=\nabla \hat{z}_{k} \cdot\left(\mathbf{r}_{k}-\mathbf{r}_{k-1}\right)$ and $\frac{p_{k-1}-p_{k-2}}{\left\|\mathbf{r}_{k-1}-\mathbf{r}_{k-2}\right\|}=\nabla \hat{z}_{k} \cdot\left(\mathbf{r}_{k-1}-\right.$ $\mathbf{r}_{k-2}$ ).

(2) The robot moves in the opposite direction of the estimated gradient $\dot{\mathbf{r}}_{k}=-\nabla \hat{z}_{k}$ or uses other strategies to reduce the measured field value. Then the robot takes a new measurement $p_{k+1}$.

(3) If $(1+\varepsilon) p_{k} \geq \bar{p}_{k}+\varepsilon z_{\min }$, the robot checks the value of $p_{k+1}-p_{k}$. If $p_{k+1}-p_{k} \leq-\rho p_{k}+\rho z_{\min }$, then it keeps individual exploration. Otherwise, it switches to cooperative exploration. If for all $k>0, p_{k+1}-p_{k} \leq-\rho p_{k}+\rho z_{\min }$ whenever $(1+\varepsilon) p_{k} \geq \bar{p}_{k}+\varepsilon z_{\min }$, the robot will converge to the local minimum $z_{\min }$ according to Corollary 1 .

In the experiment, we have no knowledge about the field minimum. Therefore, we approximate $\rho$ and $\varepsilon$ by 0 , so that the condition $p_{k+1}-p_{k} \leq-\rho p_{k}+\rho z_{\min }$ whenever $(1+\varepsilon) p_{k} \geq \bar{p}_{k}+\varepsilon z_{\text {min }}$ is simplified to $p_{k+1}-p_{k}<0$ whenever $p_{k}>\bar{p}_{k}$. The physical meaning of this condition is as follows. When the measurement at step $k$ is greater than $\bar{p}_{k}$, we check whether the next measurement $p_{k+1}$ is larger than $p_{k}$, if so, the agent is not moving in the right direction, therefore, it switches to cooperative exploration. Actually, this is the condition in the Razumikhin theorem on stability, but not asymptotic stability. Because of the disturbances and noises in the field and measuring process, we still observe the convergence to the field minimum, which is not surprising since the conditions in the Razumkhin Theorem is sufficient but not necessary.

\section{B. Cooperative exploration}

In the cooperative exploration phase, we treat the entire formation formed by all the robots as a "super-agent". Sensor readings from agents will be combined by a cooperative $H_{\infty}$ filter so that the noise level will be reduced comparing to the individual exploration phase.

Based on the relative distance of each robot to the formation center, we can define matrices $A_{k}=$ 
$\left(\begin{array}{cc}1 & \left(\mathbf{r}_{c, k}-\mathbf{r}_{c, k-1}\right)^{T} \\ 0 & \mathbf{I}_{3 \times 3}\end{array}\right), C_{k}$ as a $N \times 3$ matrix with its $i$ th row defined by $\left[1,\left(\mathbf{r}_{i, k}-\mathbf{r}_{c, k}\right)^{T}\right]$ and $D_{k}$ as the $N \times 4$ matrix with the $i$ th row vector defined by the Kronecker product $\frac{1}{2}\left(\left(\mathbf{r}_{i, k}-\mathbf{r}_{c, k}\right) \otimes\left(\mathbf{r}_{i, k}-\mathbf{r}_{c, k}\right)\right)^{T}$, where $\mathbf{r}_{i, k}$ and $\mathbf{r}_{c, k}$ denote the position of the $i$ th robot and the position of the formation center at step $k$ respectively. If we choose the state to be $\mathbf{s}_{k}=\left(z_{c, k}, \nabla z_{c, k}^{T}\right)^{T}$, then we can write down the state equation and measurement equation as

$$
\begin{array}{r}
\mathbf{s}_{k+1}=A_{k} \mathbf{s}_{k}+\mathbf{h}_{k}+\mathbf{w}_{k}, \\
\mathbf{p}_{k}=C_{k} \mathbf{s}_{k}+D_{k} \vec{H}_{c, k}+\mathbf{v}_{k},
\end{array}
$$

where $\mathbf{w}_{k}$ is a $3 \times 1$ state noise vector, $\mathbf{v}_{k}$ is a $N \times 1$ measurement noise vector and $\vec{H}_{c, k}$ is the estimate of the field Hessian $H_{c, k}$ in a vector form. $\mathbf{h}_{k-1}$ is defined as $\left(0, E\left[H_{c, k}\left(\mathbf{r}_{c, k}-\mathbf{r}_{c, k-1}\right)\right]^{T}\right)^{T}$.

Given the state equation (4) and measurement equation (5), a cooperative $H_{\infty}$ filter is constructed to provide the estimates of the field value and the field gradient at the formation center. The equations of the cooperative $H_{\infty}$ filter are as follows.

$$
\begin{aligned}
& S_{k}=P_{k}^{-1}-\frac{1}{\gamma^{2}} Q_{k}+C_{k}^{T} V_{k}^{-1} C_{k}, \\
& K_{k}=S_{k}^{-1} C_{k}^{T} V_{k}^{-1}, \\
& \hat{s}_{k+1}=A_{k} \hat{s}_{k}+\mathbf{h}_{k}+A_{k} K_{k}\left(\mathbf{p}_{k}-C_{k} \hat{s}_{k}-D_{k} H_{k}\right), \\
& P_{k+1}=A_{k} S_{k}^{-1} A_{k}^{T}+W_{k},
\end{aligned}
$$

where $\gamma$ is the noise attenuation level. $Q_{k}, W_{k}$ and $V_{k}$ are the weights of the noises. The following assumptions are made: (A1) Since the noise properties of the field are unknown, we can select $Q_{k}=\sigma_{1}^{2} I, W_{k}=\sigma_{2}^{2} I$ and $V_{k}=\sigma_{3}^{2} I$ where $I$ is the identity matrix. (A2) When $k \rightarrow \infty$, the formation is stabilized. Therefore, $C_{k}$ will converge to a constant matrix $C_{\infty}$ with its $i$ th row defined by $\left[1,\left(\mathbf{r}_{i}-\mathbf{r}_{c}\right)^{T}\right]=\left[1, \mathbf{d}_{i}^{T}\right]$, where $\mathbf{d}_{i}=\left[\begin{array}{ll}d_{i 1} & d_{i 2}\end{array}\right]^{T}$ in 2D. (A3) As $k \rightarrow \infty$, the formation will either reach a local minimum and stay there, or wander around a level curve. In either cases, the matrix $A_{k}$ can be approximated by $A_{\infty}=I$.

We have derived sufficient conditions for the feasibility and convergence of the cooperative $H_{\infty}$ filter regarding the noise attenuation level, the formation size and the number of the robots in [7] for general cases. In the experiments, we arrange the robots to be in a symmetric formation so that $\left|\mathbf{d}_{i, k}\right|=a$, where $a$ is a constant. Denote the angle between the vector $\mathbf{d}_{1, k}$ and the inertial frame as $\theta$, and the angle between $\mathbf{d}_{i, k}$ and $\mathbf{d}_{1, k}$ as $\theta_{i}=\frac{2 \pi}{N}(i-1)$. Then we can obtain $\mathbf{d}_{i, k}=a\left(\cos \left(\theta_{i}+\theta\right), \sin \left(\theta_{i}+\theta\right)\right)^{T}$. We have the following proposition for symmetric formations.

Proposition 2: For a symmetric formation under assumptions (A1)-(A3), the solution $P_{k}$ of the Riccati equation (9) at every step $k$ is feasible and converges to the stabilizing solution $P^{s}$ when $k \rightarrow \infty$ if

(1) the attenuation level $\gamma$ satisfies:

$$
\gamma^{2}>\max \left(\frac{\sigma_{1}^{2} \sigma_{3}^{2}}{N}, \frac{2 \sigma_{1}^{2} \sigma_{3}^{2}}{a N}\right) .
$$

(2) $0<P_{0}<P^{s}$ where

$$
\begin{gathered}
P^{s}=\frac{1}{2} \operatorname{diag}\left(\sigma_{2}\left(\sigma_{2}^{2}+\frac{4 \gamma^{2}}{\sigma_{3}^{-2} \gamma^{2} N-\sigma_{1}^{2}}\right)^{\frac{1}{2}}+\sigma_{2}^{2},\right. \\
\sigma_{2}\left(\sigma_{2}^{2}+\frac{8 \gamma^{2}}{a \sigma_{3}^{-2} \gamma^{2} N-2 \sigma_{1}^{2}}\right)^{\frac{1}{2}}+\sigma_{2}^{2}, \\
\left.\sigma_{2}\left(\sigma_{2}^{2}+\frac{8 \gamma^{2}}{a \sigma_{3}^{-2} \gamma^{2} N-2 \sigma_{1}^{2}}\right)^{\frac{1}{2}}+\sigma_{2}^{2}\right) .
\end{gathered}
$$

Proof: The difference Riccati equation (DRE) (9) can be written as

$P_{k+1}=A_{k} P_{k} A_{k}^{T}-A_{k} P_{k}\left[\left(C_{k}^{T} V_{k}^{-1} C_{k}-\frac{1}{\gamma^{2}} Q_{k}\right)^{-1}+P_{k}\right]^{-1} P_{k} A_{k}^{T}+W_{k}$

As $k \rightarrow \infty$, we plug $A_{\infty}, Q_{\infty}, W_{\infty}$ and $V_{\infty}$ into the above equation and drop the subscripts $\infty$. After rearranging the terms, we can get $P^{2}-\sigma_{2}^{2} P-\sigma_{2}^{2} X=0$, where $X=\left(\sigma_{3}^{-2} C^{T} C-\right.$ $\left.\sigma_{1}^{2} \gamma^{-2} I\right)^{-1}$. Based on the symmetry, we can verify the following relationships,

$$
\begin{gathered}
\sum_{i=1}^{N} \mathbf{d}_{i}=a \sum_{i=1}^{N}\left(\cos \left(\theta_{i}+\theta\right), \sin \left(\theta_{i}+\theta\right)\right)^{T}=\mathbf{0}, \\
\sum_{i=1}^{N} d_{i 1}^{2}=a^{2} \sum_{i=1}^{N} \cos ^{2}\left(\theta_{i}+\theta\right)=\frac{1}{2} a^{2} N, \\
\sum_{i=1}^{N} d_{i 2}^{2}=a^{2} \sum_{i=1}^{N} \sin ^{2}\left(\theta_{i}+\theta\right)=\frac{1}{2} a^{2} N, \\
\sum_{i=1}^{N} d_{i 1}^{2} d_{i 2}^{2}=a^{2} \sum_{i=1}^{N} \cos \left(\theta_{i}+\theta\right) \sin \left(\theta_{i}+\theta\right)=0 .
\end{gathered}
$$

Therefore, we can get $X=\operatorname{diag}\left(\sigma_{3}^{-2} N-\sigma_{1}^{2} \gamma^{-2}, \frac{1}{2} a \sigma_{3}^{-2} N-\right.$ $\left.\sigma_{1}^{2} \gamma^{-2}, \frac{1}{2} a \sigma_{3}^{-2} N-\sigma_{1}^{2} \gamma^{-2}\right)^{-1}$. We have proved in [7] that if $X>0$ and $P_{0}<P^{s}$, then the solution to the Ricatti equation (9) at every step is feasible and converges to the steady state solution $P^{s}$. In order to obtain $X>0$, we should have $\gamma^{2}>\max \left(\frac{\sigma_{1}^{2} \sigma_{3}^{2}}{N}, \frac{2 \sigma_{1}^{2} \sigma_{3}^{2}}{a N}\right)$. We can also calculate that $P^{s}=\frac{1}{2}\left(\sigma_{2}^{2} I+\left(\sigma_{2}^{4} I+4 \sigma_{2}^{2} X\right)^{\frac{1}{2}}\right)$. Substitute $X$ into the above equation, we can obtain equation (11) in Proposition 2.

From the relationships (10) and (11), we can see that to achieve a lower noise attenuation level $\gamma$ and a smaller steady state error bound $P_{S}$, we can increase the number of robots and enlarge the size of the formation. It is understandable since the more robots deployed, the more measurements processed in the cooperative $H_{\infty}$ filter at each step, which provides more information. Also, the performance of the robots becomes similar to a single robot if the robots get too close to each other. $\gamma$ and $P^{s}$ also depend on the noise strength in the field and in the measurement process.

After obtained the field value and the gradient at the formation center, we direct the formation to follow the opposite direction of the gradient: $\dot{\mathbf{r}}_{c, k}=-\nabla z_{c, k}$. The formation is described using Jacobi vectors and the readers can refer to [2] for the details of the formation control.

If the field noise reduces to the extent that the robots can explore the field by their own, they don't have to keep cooperation. We utilize the signal-to-noise ratio (SNR) to serve as the switching condition from cooperative exploration 
to individual exploration. If we define the signal-to-noise ratio obtained by the $i$ th robot at the step $k$ as

$$
\beta_{i, k}=10 \lg \sum_{\xi=0}^{r} \frac{\hat{p}_{i, k-\xi}^{2}}{\left(p_{i, k-\xi}-\hat{p}_{i, k-\xi}\right)^{2}}, i=1, \cdots, N
$$

where $\hat{p}_{i, k}^{2}$ is the estimated field noise obtained by $\hat{p}_{i, k}=$ $z_{c, k}+\left(\mathbf{r}_{i, k}-\mathbf{r}_{c, k}\right)^{T} \nabla z_{c, k}$. Then we have the following algorithm for the robots to decide when to switch from cooperative exploration to individual exploration.

Algorithm 2: Define the average signal-to-noise ratio at time step $k$ as $\bar{\beta}_{k}=\frac{1}{N} \sum_{i=1}^{N} \beta_{i, k}$. Suppose that at time $T_{s}$, the robots switch to cooperative exploration. Then for $k>T_{s}+r$, the cooperative robots switch back to individual exploration if $\bar{\beta}_{k}>\mu \bar{\beta}_{T_{s}+r}$ where $\mu>1$ is a constant.

\section{EXPERIMENTS}

In this section, we describe the multi-robot exploration test-bed and discuss the experiments for the switching strategy implemented on the test-bed.

\section{A. Experimental test-bed}

Fig. 1 shows the experimental test-bed that includes several components:

1) Robots and sensors: We choose Khepera III robots to implement the switching strategy. The Khepera III robot is a round mobile robot with two differential drive wheels and a sliding support. In our experiment, we use the nine IR sensors around the robot to measure the ambient light intensity. The sensor readings are normalized to be in the range $[0,5000]$. Note that the higher the light intensity is, the lower the sensor reading is.

2) Localization system: As seen in Fig. 1, a localization system consists of a overhead camera, a camera support and a piece of software developed based on the LabVIEW vision system. The localization system is utilized for providing the positions and orientations of the robots at each time instance.

3) Central computer: A central controller is running on a central computer in the cooperative exploration phase. At each time instance, the localization system sends the new positions and orientations of the robots to the central computer. At the same time, the robots send the measurements of the light field to the central computer. Given these information, the central computer calculates the new positions and orientations of the robots at the next step and send the corresponding moving distances and turning angles back to the robots. The robots move to the new positions according to the received command. In the individual exploration phase, since the robots are searching the field on their own, the central computer does nothing but receives the positions and orientations of the robots from the localization system.

4) Light field: We use a standard $40 \mathrm{~W}$ incandescent light bulb to serve as a light source, which generates a light field that is unknown to the robots. The field is about 2.8 meters long and 1.6 meters wide. The light intensity decreases when the distance from the light source increases, which means that the location of the light bulb hosts the maximum of the intensity of the light field. Therefore, seeking for the maximum of the light field corresponds to finding the minimum of the measured field. Even though the field appears simple, we have observed large noises in measurements, which makes individual exploration difficult.

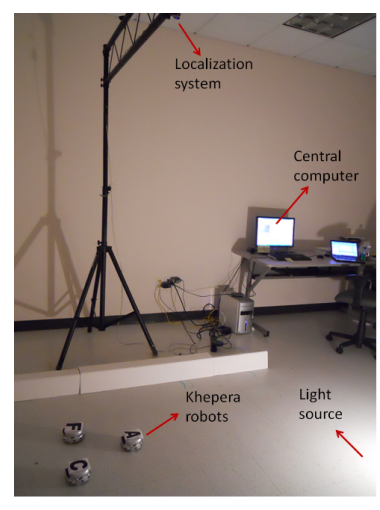

Fig. 1. The experimental setting.

\section{B. Experimental results}

We deploy three robots in the light field labeled as "A", "F" and "C" respectively. Fig. 2 shows the trajectories of the three robots when searching for the light source with the switching strategy with the memory length $r=5$. As illustrated in the figure, at first few steps, the three robots are exploring the field independently. After several steps, they realize that they can not keep individual exploration because of the field noise and measurement noise, so they switch to cooperative exploration and find the light source. The behaviors of the robots are justified by Fig. 3, in which the green, red and yellow lines indicate the measurements taken by the robot $A, F$ and $C$ respectively, and the blue line indicates the filtered field value at the formation center after they switch to cooperative exploration. The magenta vertical line shows the moment when the switching condition is violated.

In this experiment, at step $k=13$, robot $A$ detects that $p_{13}>\max _{s \in[-5,0]} p_{13+s}$. According to the switching condition from individual exploration to cooperative exploration, at step $k=14$, the robot takes a new measurement and detects that $p_{14}>p_{13}$. In this case, the switching condition in Algorithm 1 is satisfied and the robot $A$ decides to switch to cooperative exploration. It sends a switching signal to the central computer, then the central computer broadcasts a signal to all the robots. Due to the communication delay, at step $k=16$, all the robots receive the signal from the central computer and start to cooperate. The switching time $T_{s}=16$. Fig. 4 shows the average SNR $\bar{\beta}_{k}$ calculated by the central computer. At step $T_{s}+r=21$, the SNR value is 55.88, which is treated as the reference value for the switching condition from cooperative exploration to individual exploration in Algorithm 2. We set the constant $\mu=1$.4. The choice of $\mu$ is based on the experience and the performance requirements. In this experiment, since we do not control the field noise and measurement noise, therefore, the robots never detect that the 
field noise level decreases to the extent that they can switch back to individual exploration. They keep exploring the field cooperatively and locate the field minimum in around 50 steps. Other parameters in these experiments are as follows: the formation size $a=0.2 \mathrm{~m}$, the noise attenuation level $\gamma=3$, the weighting matrices $Q=I, W=0.01 I$ and $V=0.01 I$.

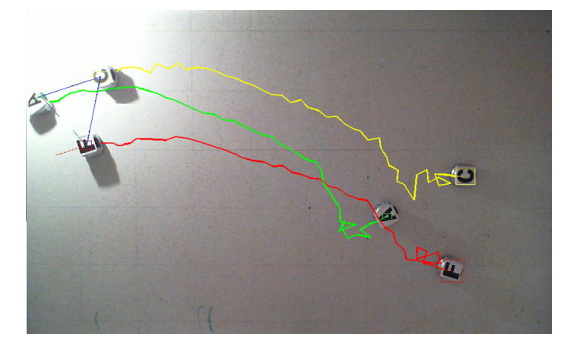

Fig. 2. Trajectories of three robots seeking for the light source with the switching strategy.

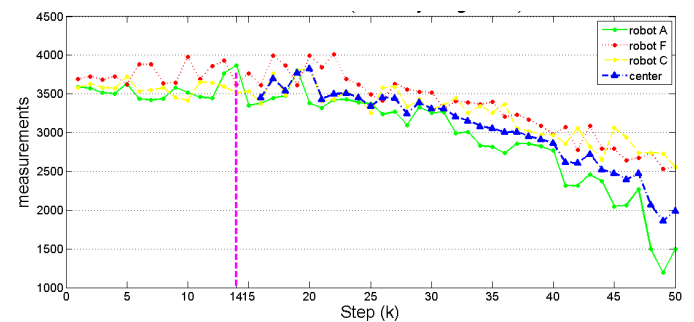

Fig. 3. Measurements when memory length is 5 . At step $k=16$, the robots switch to cooperative exploration.

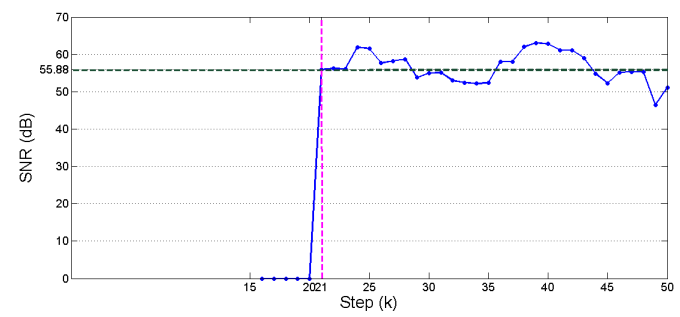

Fig. 4. Signal-to-noise ratio (SNR) when the robots are performing cooperative exploration. The memory length is 5 . At step $k=21$, the robots start to calculate the SNR.

1) Effects of the memory length $r$ : To illustrate the influence of the memory length on the exploration behavior of the robots, we conduct two additional experiments with memory lengths 10 and 20. As seen in Fig. 5 and Fig. 6, the robots with memory length 10 switch to cooperative exploration sooner and take around 60 steps to locate the light source, while the robots with memory length 20 take around 80 steps.

From the three trials with different memory lengths, we can see that the memory length $r$ plays an important role in the switching strategy. For a given field, if the noise level is high so that it is hard for the robots to find the source by themselves, the shorter the memory length is set to be, the earlier the robots realize the situation and switch to cooperative exploration. On the other hand, a longer

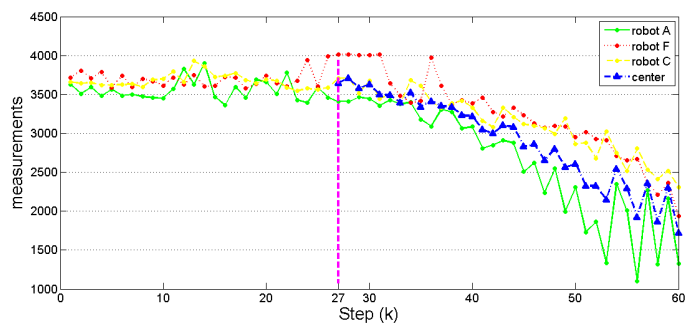

Fig. 5. Measurements when memory length is 10 . At step $k=27$, the robots switch to cooperative exploration.

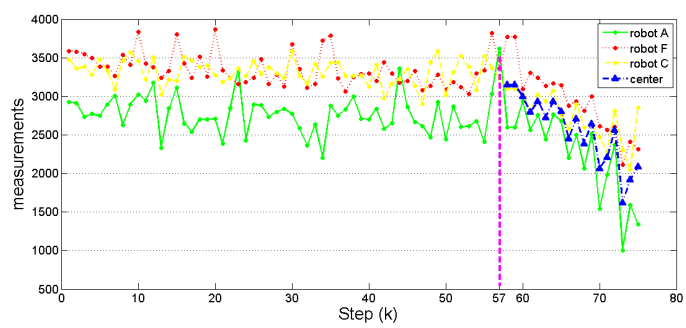

Fig. 6. Measurements when memory length is 20 . At step $k=57$, the robots switch to cooperative exploration.

memory indicates higher noise tolerance. In situations that the cooperative exploration cost is high so that it is preferable for robots to explore the field individually, longer memory lengths give more chances to the robots to explore the field by their own. Fig. 7 shows the measurements taken by one robot in another trial where the memory length is set to be 60, which is long enough for the robot not to switch to cooperative exploration. We can see from the figure that even though the measurements are noisy, since the switching condition is not satisfied with $r=60$, the robot is able to find the light source finally after around 100 steps. It is clear that cooperative exploration has better performance. However, we would like to emphasis that it may not be desirable to perform cooperative exploration from the very beginning since if the field contains very little noise, then individual exploration may be good enough.

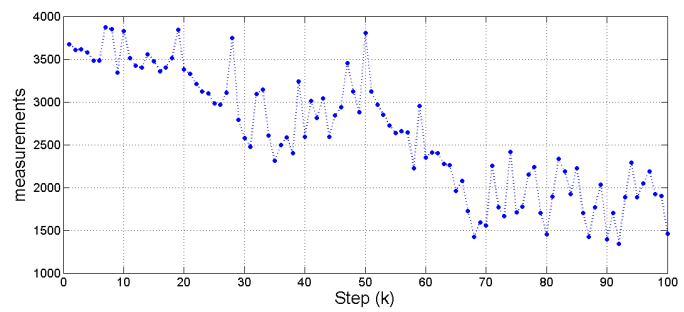

Fig. 7. Measurements taken by one robot with memory length $r=60$ but doesn't switch.

2) Effects of the noise attenuation level $\gamma$ : Fig. 8 illustrates the trace of the error bound $P_{k}$ of the $H_{\infty}$ filter associated with different noise attenuation levels when the robots are in the cooperative exploration phase. In these experiments, we also set $a=0.2 \mathrm{~m}, Q=I, W=0.01 I$ and $V=$ $0.01 I$. Given the parameters and from the sufficient conditions (10), we can calculate that if $\gamma^{2}>\max \left(\frac{\sigma_{1}^{2} \sigma_{3}^{2}}{N}, \frac{2 \sigma_{1}^{2} \sigma_{3}^{2}}{a N}\right)=$ 
$\max \left(\frac{0.01}{3}, \frac{2 \times 0.01}{0.2 \times 3}\right)=0.033$, which means $\gamma>0.1826$, the cooperative $H_{\infty}$ filter will converge. In Fig. 8, we can see that, when $\gamma>0.1826$, the noise bound $P_{k}$ converges to a steady state value $P^{s}$. Actually, since (10) is the sufficient condition, when $\gamma<0.1826$, the cooperative $H_{\infty}$ filter may converge as well. We have tested that when $\gamma>0.045$, the cooperative $H_{\infty}$ filter converges. Only when $\gamma<0.045$, the cooperative $H_{\infty}$ filter becomes unstable.

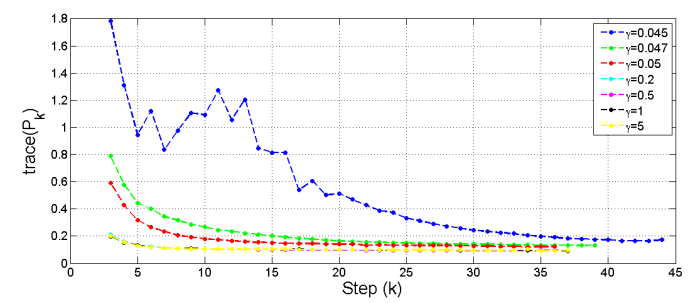

Fig. 8. Trace $\left(P_{k}\right)$ when the noise attenuation level of the $H_{\infty}$ filter $\gamma$ varies.

3) Effects of the formation size a: Fig. 9 illustrates the traces of the error bound $P_{k}$ of the $H_{\infty}$ filter associated with different formation sizes when the robots are in the cooperative exploration phase. In these experiments, we set the noise attenuation level $\gamma=3$, the weighting matrices $Q=I, W=0.01 I, V=0.01 I$. Equation (11) also indicates that as the formation size $a$ increases, $P^{s}$ reduces. We can clearly see the tendency in Fig. 9.

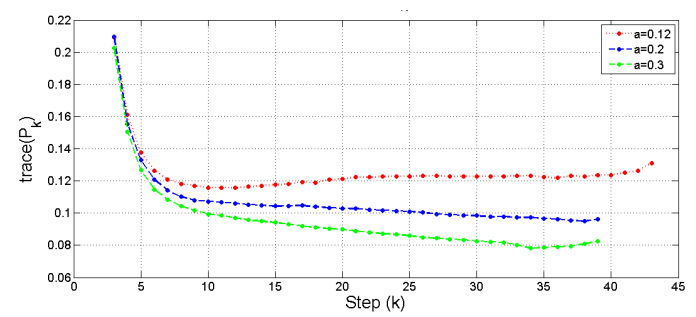

Fig. 9. Trace $\left(P_{k}\right)$ when the distance between each pair of the robots varies.

Description of the attached video: A video is submitted as an attachment of the paper. The video contains two parts. The first part demonstrates three Khepera III robots seeking for a light source with the switching strategy. In the video, the measurements and the estimates of the field value from the cooperative $H_{\infty}$ filter are shown simultaneously with the movements of the robots. The moving magenta vertical lines in the measurement figures indicate the memory lengths of the robots, and the color changes when a robot detects that the switching conditions are satisfied. In the experiment shown in the video, the robot " $F$ " first decides to switch to cooperative exploration. In the second part, we demonstrate and compare the exploration behaviors of the robots associated with different memory lengths. Under identical settings, the robots with shorter memory lengths switch to cooperative exploration sooner.

\section{CONCLUSION}

We introduce a strategy for a group of robots to locate a local minimum in an unknown field efficiently by switching between individual exploration and cooperative exploration. The strategy is implemented on a multi-robot test-bed. We have observed from the experiments that (1). Given a noisy field, longer memory lengths have higher tolerance of noises while shorter memory lengths help the robots realize that they can not finish the task earlier. (2). Our switching strategy is robust to realistic communication delays. (3). The theoretical bound of the noise attenuation level $\gamma$ is verified in the experiments. And the bound is conservative. (4). The error bound $P^{s}$ of the cooperative $H_{\infty}$ filter reduces when the formation size increases. In our future work, we will compare the performance of the switching strategy with the biological data collected from fish groups.

\section{REFERENCES}

[1] P. Ogren, E. Fiorelli, and N. E. Leonard, "Cooperative control of mobile sensor networks: Adaptive gradient climbing in a distributed environment," IEEE Transactions on Automatic Control, vol. 49, no. 8, pp. 1292-1302, 2004.

[2] F. Zhang and N. E. Leonard, "Cooperative control and filtering for cooperative exploration," IEEE Transactions on Automatic Control, vol. 55 , no. 3 , pp. $650-663,2010$.

[3] R. Graham and J. Cortes, "Cooperative adaptive sampling via approximate entropy maximization," in Proc. of 48th IEEE Conf. on Decision and Control, Shanghai, China, 2009, pp. 7055-7060.

[4] Z. Jin and A. L. Bertozzi, "Environmental boundary tracking and estimation using multiple autonomous vehicles," in Proc. of 46th IEEE Conf. on Decision and Control, 2007, pp. 4918-4923.

[5] R. Bachmayer and N. E. Leonard, "Vehicle networks for gradient descent in a sampled environment," in Proc. of 41st IEEE Conf. on Decision and Control. IEEE, 2002, pp. 113-117.

[6] C. Torney, Z. Neufeld, and L. Couzin, "Context-dependent interaction leads to emergent search behavior in social aggregates," Proc. of the National Academy of Sciences, vol. 106, no. 52, pp. 22 055-22060, 2009.

[7] W. Wu and F. Zhang, "A switching strategy for robust cooperative exploration," in Proc. of 2010 IEEE Conf. on Decision and Control, 2010, pp. 5493-5498.

[8] C. Manzie and M. Krstic, "Extremum seeking with stochastic perturbations," IEEE Transactions on Automatic Control, vol. 54, pp. 580-585, 2009.

[9] J. Cochran and M. Krstic, "Nonholonomic source seeking with tuning of angular velocity," IEEE Transactions on Automatic Control, vol. 54, no. 4, pp. 717-731, 2009.

[10] M. Stankovic and D. Stipanovic, "Extremum seeking under stochastic noise and applications to mobile sensors," Automatica, vol. 46, pp. 1243-1251, 2010.

[11] K. Gu, V. L. Kharitonov, and J. Chen, Stability of Time-Delay Systems. Boston: Birkhäuser, 2003.

[12] S. Elaydi and S. Zhang, "Stability and periodicity of difference equations with finite delay," Funkcialaj Ekvacioj, vol. 37, pp. 401413, 1994.

[13] D. Simon, Optimal State Estimation. Hoboken, New Jersey: WileyInterscience, 2006.

[14] K. M. Nagpal and P. P. Khargonekar, "Filtering and smoothing in a $H_{\infty}$ setting," IEEE Transactions on Automatic Control, vol. 36, pp. 152-166, 1991.

[15] I. Yaesh and Y. Theodor, " $H_{\infty}$ optimal estimation: a turtorial," in Proc. of 31st IEEE Conf. on Decision and Control, Tucson, Arizona, 1992, pp. 2278-2286.

[16] L. Cremean, W. B. Dunbar, D. Gogh, J. Hickey, E. Klavins, J.Meltzer, and R. M. Murray, "The Caltech multi-vehicle wireless testbed," in Proc. of 41st IEEE Conf. on Decision and Control, 2002, pp. 86-88.

[17] N. Michael, J. Fink, and V. Kumar., "Experimental testbed for large multi-robot teams: Verification and validation," IEEE Robotics and Automation Magazine, vol. 15, no. 1, pp. 53-61, 2008.

[18] A. Joshi, T. Ashley, Y. Huang, and A. L. Bertozzi, "Experimental validation of cooperative environmental boundary tracking with onboard sensors," in Proc. of 2009 American Control Conference, St. Louis, MO, 2009, pp. 2630-2635. 